\title{
NFAT5 moves to Fat City
}

\author{
Friedrich C. Luft $^{1}$
}

Published online: 13 August 2016

(C) Springer-Verlag Berlin Heidelberg 2016

Fat City, a term of endearment for the land of milk-and-honey, is a 1972 American neo-noir boxing drama film directed by the legendary John Huston. Susan Tyrrell received a Best Supporting Actress Oscar nomination as the alcoholic, world-weary Oma. So it is for the Nuclear Factor of Activated T Cells-5 (NFAT5), the world-weary transcription factor that has something for nearly everybody.

NFAT5, also known as tonicity-responsive enhancer binding protein (TonEBP), is a member of the nuclear-factoractivated T-cell transcription-factor family [1]. NFAT5 was initially identified in the hypertonic renal inner medulla, where the protein orchestrates a genetic program to maintain cellular homeostasis [2]. However, NFAT5 plays a more diverse functional role, including a hardly appreciated function in blood pressure regulation and in the development of autoimmune diseases. Despite the growing significance of NFAT5 in physiology and diseases, our understanding of how NFAT5 is regulated is limited. Furthermore, how changes in tonicity are converted into functional outputs via NFAT5 remains elusive. More than a dozen protein kinases have been identified that contribute to tonicity-dependent regulation of NFAT5 [3]. Hypertonicity activates NFAT5 by increasing its nuclear localization and by transactivating its activity in the early phase and protein abundance in the late phase of activation. The inhibition of NFAT5 by hypotonicity features a decrease in nuclear NFAT5 expression.

Recent evidence indicates that NFAT5 is not solely regulated by tonicity, but instead that NFAT5 can be stimulated by

Friedrich C. Luft

friedrich.luft@charite.de

1 Experimental and Clinical Research Center, Max-Delbrück Center for Molecular Medicine and Charité Medical Faculty, Lindenberger Weg 80, 13125 Berlin, Germany various tonicity-independent mechanisms in both hypertonic and isotonic tissues [4]. Cytokines, growth factors, receptor and integrin activation, contractile agonists, ions, and reactive oxygen species have all been implicated in the positive regulation of NFAT5 expression and activity in diverse cell types, notably immune cells [5]. These data demonstrate that tonicity-independent stimulation of NFAT5 is important for various tissue-specific functions, such as enhanced cell survival, migration, proliferation, vascular remodeling, invasion, and angiogenesis.

In this issue of $\mathrm{J}$ Mol Med, $\mathrm{Li}$ et al. demonstrate that NFAT5 can bind to a TGGAAGCGTTC consensus sequence in the gene (CACNAIC) encoding the calcium channel, voltage-dependent, L type, alpha $1 \mathrm{C}$ subunit and activates the transcription of the L-type calcium channel (LTCC) [6]. The investigators performed a comparative genomic study on $5-\mathrm{kb}$ promoter regions of the CACNA1C gene across eight vertebrate species, and showed that six factors were developmentally regulated with the expression of cacnalc in a mouse P19cl6 in vitro cardiomyocyte differentiation model. Furthermore, they demonstrated the conserved nature of this interaction by using a morpholino-mediated knockdown of $n f a t 5$ in zebrafish. The knockdown prohibited the expression of cacnalc in the fish embryos and resulted in a non-contractile ventricle, while over-expression of either cacnalc or $n f a t 5$ rescued this impaired phenotype. Thus, the authors demonstrated convincingly that CACNA1C expression initiated by NFAT5 is essential for cardiac electrophysiological development and maturation. Furthermore, NFAT5-regulated CACNA1C expression was evolutionarily conserved. However, there are numerous unanswered questions.

The burning question is, "what triggers NFAT5 to initiate regulation of CACNAIC?" CACNA1C, as the authors point 
out, is clearly important to the heart. Nonetheless CACNA1C has pivotal functions elsewhere. Could it be that this NFAT5 regulation of $C A C N A 1 C$ is important throughout the life of the organism? As we found above, the signals to activate NFAT5 are many; the first found was an osmotic stimulus [2, 4]. A critical question is, "what drives the mechanisms of activation here?" Since cytokines, growth factors, receptor and integrin activation, contractile agonists, ions, and reactive oxygen species have all been implicated in the up regulation of NFAT5 expression and activity in diverse cell types, could we imagine that one of these nonosmotic stimuli activates NFAT5 to influence $C A C N A 1 C$ expression [5]? Another question regards the relevance of $C A C N A 1 C$ regulation for long-term homeostasis. The LTCC is well known. Expression in vascular smooth muscle cells (VSMC) is fairly well worked out. The channel's role in regulation cannot be disputed because socalled calcium channel blockers are a mainstay of antihypertensive treatment [7].

The authors gave us no suggestions as to what could have stimulated NFAT5 to regulate CACNA1C in their study or in their results in Zebra fish. Any of the cytokines, reactive oxygen species, or other molecules could have influenced NFAT5 activation. However, could it have been a stimulus initiated by osmolal differences? In mammals, aside from the renal medulla, textbook teaching has concluded that osmolality throughout the extracellular and intracellular space is about the same. Perturbations would result in shifts of water from one compartment to the other. Titze et al. have drawn attention to subtle osmolal differences engendered by proteoglycans [8]. They found that sodium was stored in a non-osmotically active state, largely in skin where there are large quantities of glycosaminoglycans. Glycosaminoglycans exert strong negative charges, amenable to sodium binding in substantial quantities. They showed that a high-salt diet in rats led to interstitial hypertonic sodium accumulation in skin, resulting in increased density and hyperplasia of the lymphcapillary network [9]. The mechanisms underlying these effects on lymphatics involve activation of NFAT5 in mononuclear phagocyte system (MPS) cells infiltrating the interstitium of the skin. NFAT5 bound to the promoter of the gene encoding vascular endothelial growth factor-C (VEGF-C, encoded by $V e g f c$ ) and caused VEGF-C secretion by macrophages. MPS cell depletion or VEGF-C trapping by soluble VEGF receptor-3 blocked VEGF-C signaling, augments interstitial hypertonic volume retention, decreases endothelial nitric oxide synthase expression and elevated blood pressure in response to high salt diet. The data showed that NFAT5-VEGF-C signaling in MPS cells is a major determinant of extracellular volume and blood pressure homeostasis and identified VEGF-C as an osmosensitive, hypertonicity-driven gene intimately

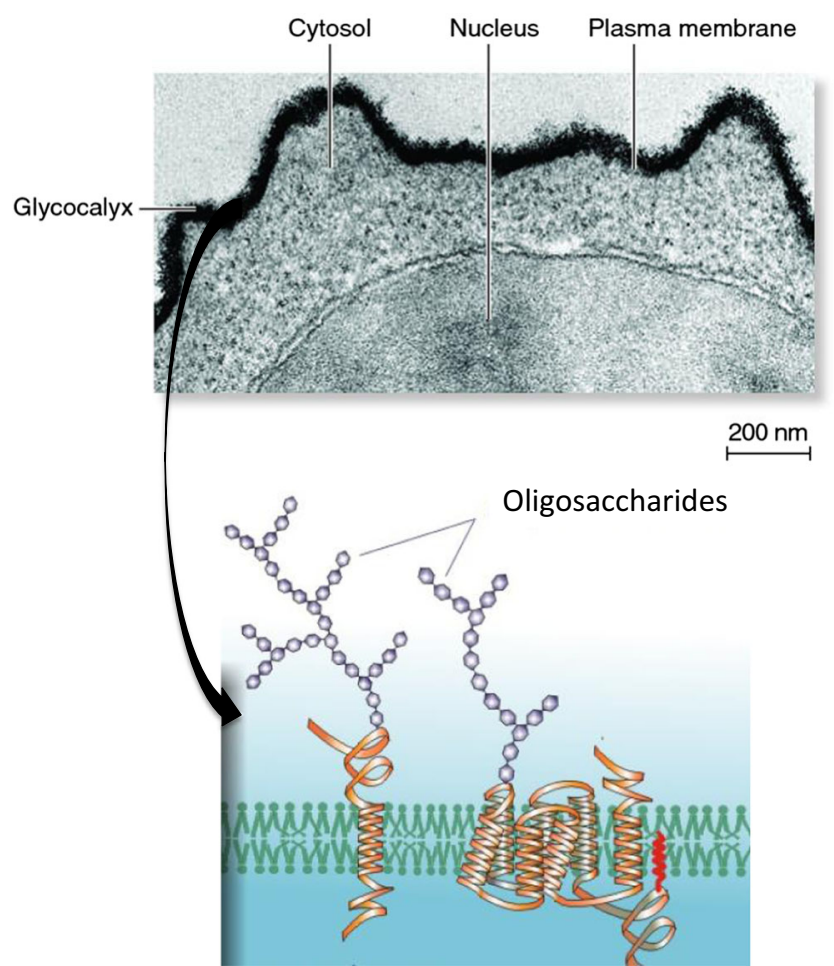

Fig. 1 a Electron micrograph of the gycocalyx. b Structural schematic model through the cell membrane. Image and illustration were from http://www.bing.com/images/search

involved in salt-induced hypertension. Nonetheless, a clear-cut explanation for increased vasoconstriction and a higher peripheral vascular resistance was not shown.

A glycocalyx (Fig. 1) resides on most cells and is luxurious, not only in skin but also on all endothelial surfaces as well as in the areolar tissue surrounding blood vessels. Could an osmolal interface exist at this site to activate NFAT5 in cells harboring $C A C N A 1 C$ [10]? To test such a notion with precision, electron microprobe analysis would have to be performed by energy-dispersive X-ray spectroscopy, as we described in an earlier study [11]. Such studies are technically demanding but possible. NFAT5 activation through osmolal mechanisms could conceivably be responsible for the local effects observed by the authors.

Respectfully,

Friedrich C. Luft

\section{References}

1. Cheung CY, Ko BC (2013) NFAT5 in cellular adaptation to hypertonic stress - regulations and functional significance. J Mol Signal 8:5

2. Miyakawa H, Woo SK, Dahl SC, Handler JS, Kwon HM (1999) Tonicity-responsive enhancer binding protein, a rel-like protein that stimulates transcription in response to hypertonicity. Proc Natl Acad Sci U S A 96:2538-2542 
3. Zhou X (2016) How do kinases contribute to tonicity-dependent regulation of the transcription factor NFAT5? World J Nephrol 5: 20-32

4. Halterman JA, Kwon HM, Wamhoff BR (2012) Tonicityindependent regulation of the osmosensitive transcription factor TonEBP (NFAT5). Am J Physiol Cell Physiol 302:C1-C8

5. Trama J, Lu Q, Hawley RG, Ho SN (2000) The NFAT-related protein NFATL1 (TonEBP/NFAT5) is induced upon T cell activation in a calcineurin-dependent manner. J Immunol 165:4884-4894

6. Li W, Zheng NZ, Yuan Q, Xu K, Yang F, Gu L, Zheng GY, Luo GJ, Fan C, Ji GJ et al (2016) NFAT5-mediated CACNA1C expression is critical for cardiac electrophysiological development and maturation. J Mol Med. doi:10.1007/s00109-016-1444-x

7. Bakris G, Briasoulis A, Dahlof B, Jamerson K, Weber MA, Kelly RY, Hester A, Hua T, Zappe D, Pitt B et al (2013) Comparison of benazepril plus amlodipine or hydrochlorothiazide in high-risk patients with hypertension and coronary artery disease. Am J Cardiol 112:255-259
8. Titze J, Shakibaei M, Schafflhuber M, Schulze-Tanzil G, Porst M, Schwind KH, Dietsch P, Hilgers KF (2004) Glycosaminoglycan polymerization may enable osmotically inactive $\mathrm{Na}+$ storage in the skin. Am J Physiol Heart Circ Physiol 287:H203-H208

9. Machnik A, Neuhofer W, Jantsch J, Dahlmann A, Tammela T, Machura K, Park JK, Beck FX, Muller DN, Derer W et al (2009) Macrophages regulate salt-dependent volume and blood pressure by a vascular endothelial growth factor-Cdependent buffering mechanism. Nat Med 15:545-552

10. Essin K, Welling A, Hofmann F, Luft FC, Gollasch M, Moosmang $S$ (2007) Indirect coupling between Cav1.2 channels and ryanodine receptors to generate $\mathrm{Ca} 2+$ sparks in murine arterial smooth muscle cells. J Physiol 584:205-219

11. Wiig H, Schroder A, Neuhofer W, Jantsch J, Kopp C, Karlsen TV, Boschmann M, Goss J, Bry M, Rakova N et al (2013) Immune cells control skin lymphatic electrolyte homeostasis and blood pressure. J Clin Invest 123:2803-2815 